\title{
Traditional Chinese Baduanjin Qigong for Older Adults: A Mini-Review
}

\author{
Liye $\mathrm{Zou}^{1 *}$ and Chaoyi Wang ${ }^{2}$ \\ ${ }^{1}$ Springfield, USA \\ ${ }_{2}^{2}$ Jilin University, China
}

Submission: April 01, 2017; Published: April 11, 2017

*Corresponding author: Liye Zou, 625 Newbury Street, Springfield, MA 01104; USA, Tel: 413-241-0705; Email: liyezou123@gmail.com

\section{Introduction}

Baduanjin (Ba Duan Jin) is one of the forms of traditional Qigong exercises (e.g., Taichi Quan, Wuqinxi Qigong, and Mawangdui Qigong) which was created in ancient China and has been developed by Chinese Health-Qigong association for chronic diseases prevention and treatment [1]. Baduanjin Qigong has an emphasis on a combination of physical exercise with mental focus; interaction between symmetrical physical postures and movements, meditative mind, and breathing techniques in a harmonious manner. In addition, when compared to complex and lengthy traditional Taichi form, Baduanjin Qigong is less physically and cognitively demanding, and are easier to learn and practice at home $[2,3]$. Therefore, it is very suitable for special populations such as older adults who are experiencing age-related memory decline may have difficulty executing traditional Taichi form.

Baduanjin Qigong has concentrated on cultivating internal vital energy for the aim of producing diverse health benefits according to the Chinese traditional medicine theory. To promote Baduanjin Qigong, the establishments of Health-Qigong Associations have emerged worldwide and the number of Baduanjin Qigong practitioners has grown in different regions of the globe. More and more researchers have paid attention to this phenomenon and utilized the scientific methods to investigate the effects of Baduanjin Qigong on health-related parameters in different populations. While evidence arising from these studies indicates that Baduanjin Qigong is beneficial for health, summarizing the existing literature with regard to the beneficial effects of Baduanjin Qigong is warranted and provides guideline for health professionals.

\section{Summary of Research Findings}

To learn more about how Baduanjin Qigong might be used as an intervention for older adults, a literature review was conducted focusing on the benefits of Baduanjin Qigong on sleep quality [4], balance function [5], memory function [6], and cognitive function [7], of the four articles reviewed, three utilized a randomized controlled trial, one utilized quasiexperimental study design, and all employed a longitudinal research design.

In the articles reviewed, the intervention was either Baduanjin Qigong compared with no intervention [4], or Baduanjin Qigong compared with walking exercise intervention [5] or Baduanjin Qigong compared with Tai Chi exercise intervention and no intervention [6]. Elder individuals participated in Baduanjin sessions that lasted 30-60 minutes. Overall programs lasted 12 weeks. While positive outcomes were recorded in all programs, one study specifically noted that the effect of Baduanjin on improving sleep quality of older adults started as early as 4 weeks [4], one study noted that Baduanjin on improving dynamic balance and static balance as early as 6 weeks [5] after intervention.

Participation in Baduanjin intervention resulted in significant improvement in older adults' sleep quality [4], balance function [5], memory function [6], and cognitive function [7]. After practicing Baduanjin Qigong for a period of time, older adults in the Baduanjin exercise group had significantly improved overall sleep quality, subjective sleep quality, sleep latency, sleep duration, sleep efficiency, and daytime dysfunction after 12 weeks of intervention, while those in control group showed no significant difference in sleep quality [4]. After practicing Baduanjin Qigong for 12 weeks, older adults in the Baduanjin exercise group improved their dynamic and static balance scores earlier (i.e., 6 weeks) than the older adults in the walking exercise group (i.e., 12 weeks); scores reflecting balance ability and confidence of maintaining balance in the Baduanjin exercise group increased after 12 weeks' intervention, indicating Baduanjin exercise is better than walking in improving balance ability and self-efficacy of older adults [5].

After 12 weeks' Baduanjin Qigong practice, older adults resulted in an increased memory quotient (MQ) score measured by the Wechsler Memory Scale-Chinese Revision, and no significant difference was observed in MQ between the older 
adults in the Baduanjin group and the older adults in the Tai Chi group; the resting-state functional connectivity (rs-FC) between the bilateral hippocampus and medial prefrontal cortex (mPFC) significantly increased in the Baduanjin group compared to the control group, and no significant difference between the Tai Chi and Baduanjin groups was observed, indicating a memory function improvement in Baduanjin and Tai Chi groups [6]. After 12-week Baduanjin and Tai Chi practice, both Baduanjin and Tai Chi groups demonstrated significant improvements in mental control function; subjects in the Baduanjin group showed a significant cognitive control network (CNN) rs-FC decrease in the left putamen/insula compared with subjects in the control group; significant CNN rs-FC decreases in the left superior frontal gyrus, left dorsal anterior cingulated cortex, and rostra anterior cingulated cortex in Tai Chi group compared with control group [4]. Given mental control improvement was negatively associated with CNN rs-FC changes across all subjects, the above findings demonstrate the potential of Baduanjin and Tai Chi in preventing cognitive decline.

\section{Practical Implications}

Based on the findings above, Baduanjin may be considered as an evidenced based prevention and treatment modality in several aspects, such as improving sleep quality, preventing falls, and preventing cognitive impairments. Family members are important resources for older adults' participation in Baduanjin practice, thus it is prudent that family members are involved in the program as well. We should encourage practice of Baduanjin at home and provide family with written materials or a CD that guides and supports practice at home.

\section{References}

1. Zou L, SasaKi JE, Wang H, Xiao Z, Fang Q Zhang M (2017) “A Systematic Review and Meta-Analysis Baduanjin Qigong for Health Benefits: Randomized Controlled Trials". Evidence-Based Complementary and Alternative Medicine pp: 17.

2. Zou L, Wang H, Xiao Z, Fang Q Zhang M, et al. (2017) Tai chi for health benefits in patients with multiple sclerosis: A systematic review. PloS one 12(2): e0170212.

3. Lan C, Chou SW, Chen SY, Lai JS, Wong MK (2004) The aerobic capacity and ventilatory efficiency during exercise in Qigong and Tai Chi Chuan practitioners. Am J Chin Med 32(1): 141-150.

4. Chen MC, Liu HE, Huang HY, Chiou AF (2012) The effect of a simple traditional exercise programme (Baduanjin exercise) on sleep quality of older adults: a randomized controlled trial. International Journal of nursing studies 49(3): 265-273.

5. yun Liu X, Gao J, xiang Yin B, yu Yang X, xi Bai D (2016) Efficacy of ba duan jin in improving balance: a study in chinese community-dwelling older adults. Journal of gerontological nursing 42(5): 38-46.

6. Tao J, Liu J, Egorova N, Chen X, Sun S, et al. (2016) Increased Hippocampus-Medial Prefrontal Cortex Resting-State Functional Connectivity and Memory Function after Tai Chi Chuan Practice in Elder Adults. Frontiers in aging neuroscience 8: 25.

7. Tao J, Chen X, Egorova N, Liu J, Jian Kong, et al. (2017) Tai Chi Chuan and Baduanjin practice modulates functional connectivity of the cognitive control network in older adults. Scientific Reports 7: 41581.

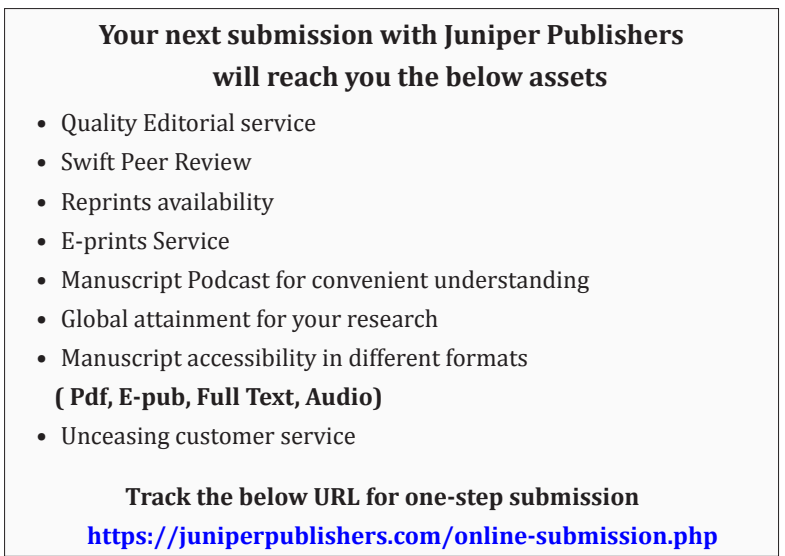

